\title{
Modificaçōes da Hemodinâmica Fetal pelo Estímulo Sonoro: Avaliação pela Dopplervelocimetria Colorida
}

\author{
Vibro-acoustic Stimulation Induced Hemodynamic Fetal \\ Changes Assessed by Color Doppler
}

\begin{abstract}
Francisco Mauad Filho, Celso Engelberto Ayres, Marcelo Braga Molinari, Rogério Lino de Matos, José Eduardo Chúfalo
\end{abstract}

\begin{abstract}
RESUMO
Objetivos: verificar se ocorrem ou não alterações hemodinâmicas na aréria cerebral média (ACM) aferido pela dopplervelocimetria colorida após realização de um estímulo sonoro.

Métodos: trinta fetos de gestantes consideradas clinicamente normais com idade gestacional igual ou superior a 28 semanas foram submetidos a um estimulo sonoro. Examinamos as alterações da velocidade sangüinea na ACM fetal por meio do indice de resistência e da freqüencia cardiaca fetal, pelo doppler colorido, antes e depois do estímulo acústico.

Resultados: a média da freqüência cardíaca fetal (FCF) antes do estímulo sonoro foi de 142,41 batimentos por minuto (bpm) com desvio padrão de 9,01 e faixa de variação de 122 a $162 \mathrm{bpm}$. Após o estímulo sonoro, a média da FCF foi de 159,44 bpm com desvio padrão de 15,49, com faixa de variação de 130 a 187 bpm (p<0,01). A média dos valores do indice de resistência (IR) da ACM fetal antes do experimento foi de 75,89\% variando entre $64 e$ 91\%. Após o estímulo acústico, a média dos valores do IR foi de 66,93\% variando entre $47 e$ $83 \%(p<0,01)$.

Conclusões: observamos que o estimulo sonoro provoca a conhecida elevação imediata $e$ significativa da FCF e a diminuição da resistência vascular cerebral, quando avaliada pelo indice de resistência na artéria cerebral média fetal.
\end{abstract}

PALAVRAS-CHAVE: Ultra-sonografia. Fisiologia fetal. Dopplervelocimetria. Sofrimento fetal.

\section{Introdução}

As condições fetais começaram a ser investigadas cientificamente somente no final do século passado, por meio de diferentes

Departamento de Ginecologia e Obstetrícia da Faculdade de Medicina de Ribeirão Preto da Universidade de São Paulo (FMRP-USP) e Escola de Ultra-sonografia e Reciclagem Médica Ribeirão Preto.

Correspondência:

Francisco Mauad Filho

Rua Casemiro de Abreu, 660 - Vila Seixas

14020-060 - Ribeirão Preto - SP procedimentos (biofísicos, bioquímicos, imunológicos, etc) $)^{7,8}$. Basicamente, eram das manifestações somáticas do concepto que se inferia algo de seu provável bem-estar. Tais estudos, no entanto, permitiam apenas uma estimativa grosseira do estado fetal e apenas durante os estágios finais da gravide $z^{12}$.

O interesse a respeito do desenvolvimento cronológico do feto, em diferentes aspectos da sua vida biológica e mental, dentro do microambiente intra-uterino, é antigo e ainda não está completamente elucidado. A vida fetal, em função de sua natureza abrigada, era considerada como 
um mundo totalmente à parte como se a vida realmente apenas começasse no ato do nascimento, se não muito depois ${ }^{17,18}$.

Até o início do século passado, consideravase que as funções sensoriais do feto eram praticamente deprimidas, senão inexistentes ${ }^{1,3}$. Desde 1925, a resposta reativa de fetos humanos a sons tem sido investigada com estimulações sonoras externas ${ }^{11}$.

Existem evidências de que o aparelho auditivo humano atinge altos níveis de maturação já durante a vida intra-uterina ${ }^{1,15}$. Observações histológicas indicam que o órgão de Corti e estruturas circunvizinhas estão anatomicamente completas em torno da $18^{\text {a }}$ semana de gestação, e que os fetos podem responder às estimulações acústicas desde as $22^{\text {a }} / 24^{\text {a }}$ semanas gestacionais e está perfeitamente estabelecido que estímulos externos podem acionar sistemas reflexos básicos no cérebro fetal ${ }^{2,3,24}$.

A observação de que estimulações sonoras induzem, sobretudo, aceleração na freqüência dos batimentos cardíacos do feto e a rápida resposta motora, motivou alguns pesquisadores a proporemnas como técnica para se avaliar as condições do feto $^{13,19,20,22,23}$. O primeiro relato na literatura sobre a capacidade do feto humano de aumentar a sua freqüência cardiaca em resposta a determinado estímulo vibratório foram feitos por Sontag e Wallace, em $1934^{25}$.

Hammacher ${ }^{16}$, aperfeiçoando a cardiotocografia, desenvolveu o primeiro monitor de uso clínico, denominado de cardiotocógrafo (CTB), que passou a ser usado para avaliar o bem-estar fetal anteparto, correlacionando diversas variáveis biofisicas fetais diante das contrações de BraxtonHicks e durante o trabalho de parto. Birnholz e Benacerraf ${ }^{6}$ aplicaram o assim denominado "electrolarynx", produzindo uma estimulação vibroacústica no abdome materno, obtendo reações fetais.

Recentemente, a ultra-sonografia mostrou os movimentos fetais desde os estágios mais iniciais da gestação, muito antes das gestantes os perceberem. Com a introdução do ultra-som doppler na obstetrícia tornou-se possível a investigação e a avaliação do estado hemodinâmico da gestante e, em particular, do concepto ${ }^{7,8,12}$. Estudos prévios demonstraram que a artéria fetal mais sensivel ao estimulo hipóxico parece ser a artéria cerebral média fetal $(\mathrm{ACM})^{21,27}$. Além disso, essa artéria desempenha um papel único no contexto da circulação cerebral, encarregando-se da irrigação da maior parte do córtex cerebral. Visando contribuir com os estudos relacionados com a fisiologia fetal, o objetivo do presente trabalho foi estudar se ocorrem ou não alterações hemodinâmicas na $\mathrm{ACM}$, aferido pela dopplervelocimetria colorida após realização de um estímulo sonoro.

\section{Pacientes e Métodos}

Estudamos de modo prospectivo a reatividade vascular de 30 fetos de gestantes atendidas na Escola de Ultra-sonografia e Reciclagem Médica Ribeirão Preto, em colaboração com a Faculdade de Medicina de Ribeirão Preto da Universidade de São Paulo (FMRP-USP), no período de fevereiro a julho de 1997.

Utilizamos como critérios de inclusão no presente estudo: presença de gestação única; idade gestacional de 28 semanas ou mais (definida pela data confiável da última menstruação e pelos exames ultra-sonográficos prévios ou atual); a presença de bolsa amniótica íntegra e a ausência de qualquer patologia materna e fetal prévia ou constatada durante a realização do experimento.

Foram oferecidas todas as informações sobre a pesquisa e consentimento por escrito foi obtido de cada gestante. Caso fosse constatado qualquer intercorrência, o exame seria interrompido imediatamente para permitir que se tomassem as medidas necessárias para garantir o bem-estar materno e fetal. Nenhuma outra estimulação, fora a do procedimento ultra-sonográfico e sonoro, foi realizada durante o experimento. Não se controlou a ingesta liquida e de alimentos, ou da temperatura ambiente nem antes nem após o exame. As pacientes foram examinadas na posição semirecostada, em mesa ginecológica, com angulação de aproximadamente $45^{\circ}$.

Todos os fetos foram estudados com o uso de um equipamento ultra-sonográfico Hitachi modelo 525, com transdutor convexo de 3,5 $\mathrm{MHz}$. Obtinha-se, primeiramente, a situação, a posição e a apresentação seguida da biometria fetal, quando era estimada a idade gestacional. Localizava-se, então, a placenta e o seu grau de senescência conforme os critérios de Grannum et al. ${ }^{14}$ e o índice do líquido amniótico (ILA).

$\mathrm{Na}$ fase seguinte, com o auxílio do doppler colorido e do power doppler, identificava-se a freqüência cardiaca fetal (FCF). Por último identificava-se o poligono de Willis e a ACM fetal, de onde era obtido o sonograma típico dessa artéria e aferido eletronicamente o seu índice de resistência (IR), antes e após o estímulo acústico. Para se estudar as artérias cerebrais, buscava-se um plano transversal, na base do crânio fetal, no nivel dos pedúnculos cerebrais. Nesse corte, a artéria carótida interna se situa logo adiante dos 
pedúnculos cerebrais, na forma de dois pontos pulsáteis, que correspondem à secção transversa. A artéria cerebral anterior se situa, por sua vez, logo adiante dessa. Por outro lado, a artéria cerebral média é visibilizada em ambos os lados das carótidas internas, apresentando uma pulsatilidade muito clara, próxima à incisura de Sylvius. Finalmente, as pulsações puntiformes das artérias cerebrais posteriores se situam pouco atrás e ao lado dos pedúnculos cerebrais.

O formato de onda espectral que é obtido com o doppler da ACM pode ser analisado e quantificado por vários métodos. Neste trabalho foi utilizado o IR, ou índice de Pourcelot, que é calculado medindose o valor do pico sistólico (S) do qual era subtraída a velocidade no fim da diástole (D),e a diferença dividida pelo valor do pico da velocidade sistólica $(\mathrm{IR}=\mathrm{S}-\mathrm{D} / \mathrm{S})$. Os IRs variam de 1,0 a 0,0 , com 1,0 indicando o máximo de resistência do fluxo que avança ou um fluxo diastólico ausente.

O estímulo sonoro foi realizado com o uso de uma fonte emissora de ondas sonoras, marca Kobo, com área de contacto de $9,62 \mathrm{~cm}^{2}(3,5 \mathrm{~cm})$, posicionada no abdome materno, próximo à cabeça fetal, padronizada para exercer pressão sonora entre 60 a $115 \mathrm{db}$ e freqüência entre 500 a 4000 Hertz, a qual era acionada manualmente durante 3 a 7 segundos. Entre dois e cinco minutos depois do estímulo sonoro era obtido novamente o sonograma e o IR da ACM. As imagens e registros da dopplervelocimetria colorida foram fotografados, permitindo assim uma análise futura, bem como a documentação do que era observado. Após cada experimento, os dados obtidos eram registrados em um protocolo padrão. Os resultados foram analisados estatisticamente utilizando-se do teste t de Student.

\section{Resultados}

A média de idade das gestantes foi de 26,3 anos, com desvio padrão (DP) de 6,78 (faixa de variação de 17 a 38 anos) e a maioria era primigesta. A média da idade gestacional foi de 33,37 semanas com DP de 4,27 . Observou-se que todos os conceptos responderam ao estímulo sonoro, assim interpretados pela presença de maior movimentação fetal e/ou pela presença de alterações da FCF e pela variação no IR, obtido na artéria cerebral fetal, desencadeados logo após o estímulo acústico ser aplicado (Figuras 1 e 2). A média da FCF antes do estimulo sonoro foi de $142,41 \mathrm{bpm}$ com desvio padrão de 9,01 e faixa de variação de 122 a 162. Após o estímulo sonoro, a média da FCF foi de 159,44 bpm com desvio padrão de 15,49 , com faixa de variação de 130 a 187 ( $p<0,01)$ (Figura 3). A média dos valores do IR da artéria cerebral média fetal (ACM) antes do experimento, foi de $75,89 \%$ (DP 7,37 ), variando entre 64 e 91\%. Após o estímulo acústico, a média dos valores do IR foi de 66,93\% (DP 9,80), variando entre 47 e $83 \%(p<0,01)$ (Tabela 1, Figura 4).

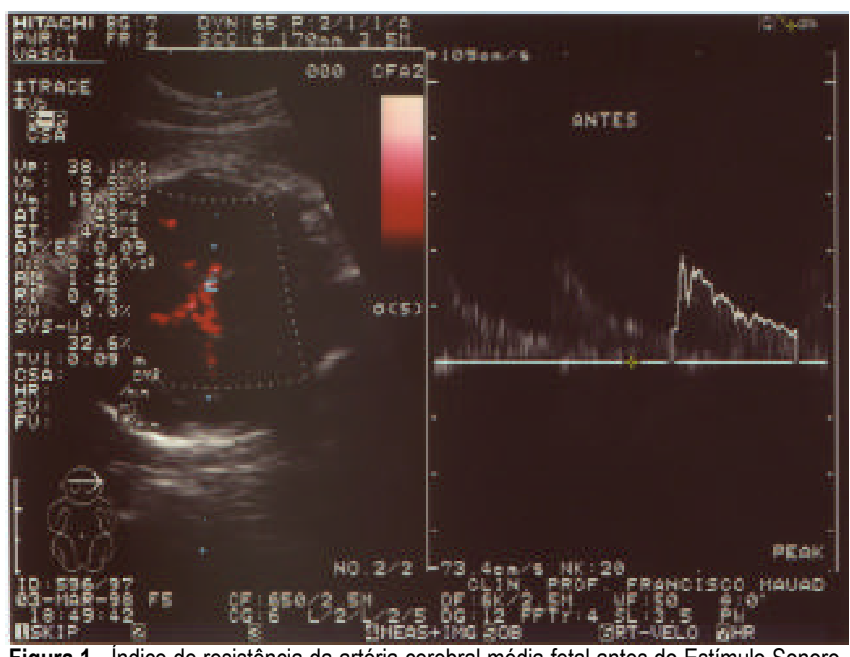

Figura 1 - Indice de resistência da artéria cerebral média fetal antes do Estímulo Sonoro.

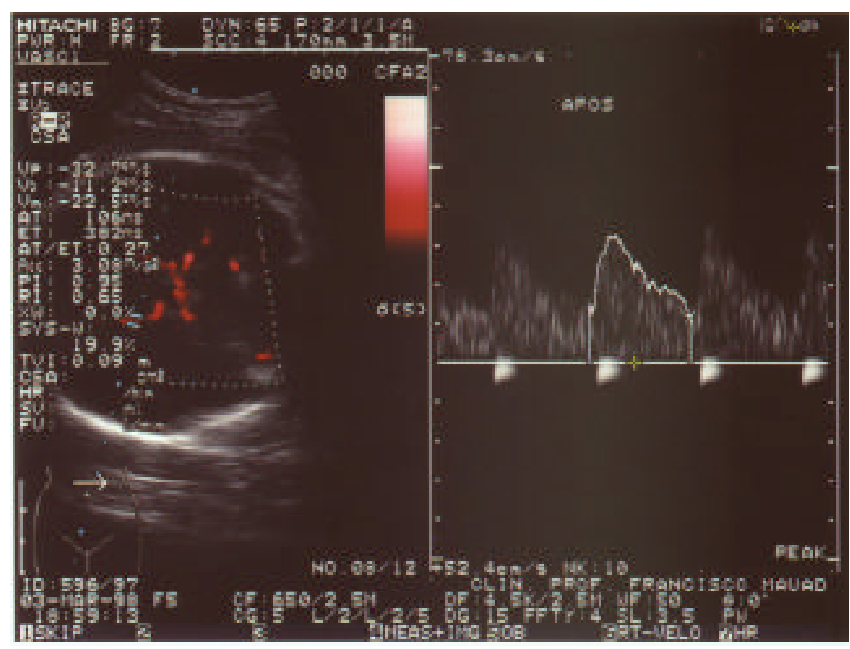

Figura 2 - Índice de resistência da arteria cerebral média fetal após o Estímulo Sonoro.

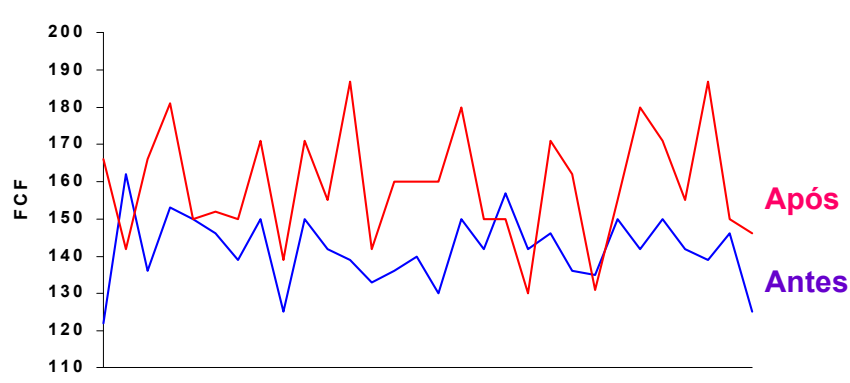

Figura 3 - Valores médios da freqüência cardiaca fetal (FCF) obtidos pelo doppler colorido antes e após o estímulo sonoro. 
Tabela 1 - Valores do índice de resistência (IR) da artéria cerebral média fetal (ACM) obtidas pelo doppler colorido antes e após um estímulo sonoro.

\begin{tabular}{|c|c|c|}
\hline Caso & IR antes & IR após \\
\hline 1 & 72 & 60 \\
\hline 2 & 84 & 81 \\
\hline 3 & 80 & 69 \\
\hline 4 & 79 & 73 \\
\hline 5 & 75 & 70 \\
\hline 6 & 79 & 70 \\
\hline 7 & 79 & 83 \\
\hline 8 & 66 & 63 \\
\hline 9 & 91 & 75 \\
\hline 10 & 88 & 72 \\
\hline 11 & 80 & 57 \\
\hline 12 & 64 & 47 \\
\hline 13 & 78 & 49 \\
\hline 14 & 86 & 68 \\
\hline 15 & 72 & 65 \\
\hline 16 & 75 & 60 \\
\hline 17 & 73 & 64 \\
\hline 18 & 78 & 66 \\
\hline 19 & 66 & 74 \\
\hline 20 & 67 & 59 \\
\hline 21 & 72 & 63 \\
\hline 22 & 73 & 71 \\
\hline 23 & 70 & 83 \\
\hline 24 & 76 & 80 \\
\hline 25 & 67 & 73 \\
\hline 26 & 88 & 72 \\
\hline 27 & 80 & 57 \\
\hline 28 & 64 & 47 \\
\hline 29 & 79 & 70 \\
\hline 30 & 91 & 75 \\
\hline Média & 75,89 & 66,93 \\
\hline Desvio padrão & 7,37 & 9,80 \\
\hline
\end{tabular}

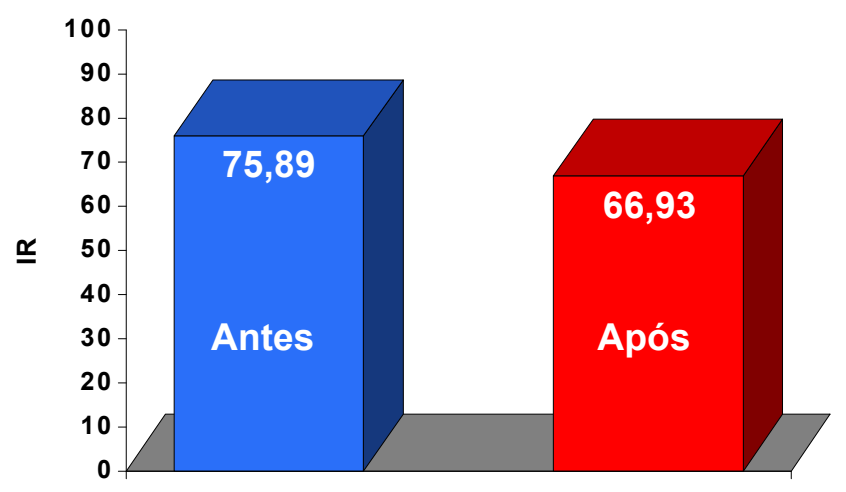

Figura 4 - Valores médios dos índices de resistência (IR) na artéria œerebral média fetal antes e após o estímulo sonoro.

\section{Discussão}

As reações do feto a estímulos sonoros e sua interpretação como indicador de bem-estar fetal têm sido observadas desde a década de vinte ${ }^{11}$. Em 1936, Sontag e Wallace ${ }^{25}$ foram os primeiros a descrever a aceleração da freqüência cardiaca fetal após um estímulo sonoro. Posteriormente, em 1947, os mesmos autores preconizaram o estudo dessa resposta cardiaca fetal como um parâmetro da reatividade fetal ${ }^{26}$. Segundo Luz et al. ${ }^{19,20}$, quando o feto se encontra em boas condições de oxigenação, verifica-se uma resposta imediata à estimulação sônica, manifestada por meio de intensa movimentação fetal e acentuada taquicardia.

Neste estudo, constatou-se à ultra-sonografia um grande repertório de complexos movimentos fetais intra-uterinos e taquicardia associada, após a estimulação acústica, de acordo com as observações citadas. A reação fetal ocorreu após um período de latência, em geral menor do que cinco segundos, sendo compativel com o descrito na literatura ${ }^{5,29}$. O curto intervalo entre a aplicação do estímulo e a obtenção da resposta, notada aqui e por outros autores ${ }^{10,13}$, sugere fortemente que o concepto responde diretamente ao estímulo, e que a resposta é reflexa e nervosa, pelo menos no que diz respeito à sua primeira fase ${ }^{28}$.

É provável que tal resposta se deva à liberação de catecolaminas pelas adrenais fetais, decorrente da situação de estresse súbita a que foi submetido. Ainda que estudos prévios sugiram que uma via auditiva funcional é capaz de conduzir impulsos nervosos apenas após a $32^{\text {a }}$ ou $34^{a}$ semana de idade gestacional ${ }^{15,17,18}$, neste experimento verificamos que todos os fetos estimulados responderam ao estímulo sonoro.

A resposta fetal provocada por um determinado estímulo pode ser analisada por meio do registro da onda elétrica cerebral específica obtida com o eletroencefalograma ${ }^{23}$ e pela cardiotocografia $(\mathrm{CTG})^{4}$. Recentemente, o estímulo sonoro passou a ser associado à cardiotocografia fetal anteparto, visando diminuir o tempo de exame e a proporção de testes não-reativos ${ }^{9,24}$. Na experiência de Behle et $\mathrm{al}^{4}$, a aplicação do estímulo sonoro é capaz de modificar os padrões fetais ditos hipoativos, analisados pela CTG de repouso, convertendo-os em 50\% dos casos. Com a utilização do ultra-som, constatamos alterações de estado e na FCF em todos os fetos. Tal enfoque permite encarar o estímulo sonoro, associado à ultra-sonografia, não apenas como complemento 
da CTG anteparto de repouso, mas como um teste original de avaliação fetal per se.

Atualmente, uma estimativa quase ideal do bem-estar fetal é possivel pela análise simultânea da variabilidade da freqüência cardíaca fetal e de diferentes parâmetros fetais obtidos com a ultrasonografia como o tônus, os movimentos respiratórios e outros, denominados em conjunto como perfil biofísico fetal. Desde 1978, a dopplervelocimetria vem sendo usada para se avaliar a circulação fetoplacentária, tendo, contudo, um papel ainda limitado na avaliação da vitalidade fetal ${ }^{12}$.

Neste trabalho, realizamos um estudo hemodinâmico da variação da freqüência cardiaca e da velocidade de fluxo sangüíneo na circulação cerebral fetal, particularmente da artéria cerebral média, por meio da dopplervelocimetria colorida. Constatamos a presença de alterações significativas no índice de resistência da $\mathrm{ACM}$, com expressivo aumento do fluxo na fase de diástole, após o estímulo sonoro. Tais achados são compativeis com a proposição enunciada por muitos autores da presença de ajustamentos circulatórios, no feto, sempre que o mesmo é ou está submetido a situações hipóxicas ou estressantes ${ }^{21,27}$. Acreditase que ocorra uma redistribuição de fluxo sangüineo fetal, o que colabora para proteger os seus órgãos vitais, tais como o cérebro e o coração.

Do exposto, ficou evidente que a estimulação sônica produz a aceleração da FCF espelhando, seguramente, uma ação sobre a função cardiaca e cerebral. Por último, o estudo doppler da ACM, após o estímulo sonoro, apresentou um freqüente aumento do fluxo diastólico, em razão da diminuição da resistência vascular, compativel com a hipótese de ajustamentos circulatórios do feto em face de diferentes situações de estresse.

Ainda que não esteja claro se o estímulo sonoro excessivo pode lesar as delicadas estruturas auditivas fetais, é certo que ele produz reações comportamentais e alterações na fisiologia fetal, eventos observados neste estudo. Novas investigações, portanto, devem ser realizadas para se avaliar a eficácia e a segurança da estimulação vibroacústica na avaliação da saúde do feto. Por outro lado, a dopplervelocimetria colorida mostrou ser uma ferramenta útil no estudo da fisiologia fetal, não-invasiva e, no futuro, poderá ser útil na avaliação dos efeitos de inúmeras drogas utilizadas na gravidez, sobre o concepto. Em resumo, os resultados do presente trabalho indicam haver alterações na reatividade vascular do feto, quando avaliados pela dopplervelocimetria colorida, no nível da artéria cerebral média fetal, após um estímulo sonoro.

\section{SUMMARY}

Purpose: to determine the possible occurrence of hemodynamic changes in the middle cerebral artery of the fetus (MCA) using color doppler after vibro-acoustic stimulation.

Methods: thirty fetuses from pregnant women considered to be clinically normal, with a gestational age of 28 weeks or more were submitted to vibro-acoustic stimulation. We examined the changes in blood flow rate in the middle cerebral artery of the fetus on the basis of resistance index (RI) and fetal heart rate (FHR) by color doppler before and after the sound stimulus.

Results: mean FHR before vibro-acoustic stimulation was 142.41 beats per minute (bpm) with a standard deviation of 9.01 and a range of 122 to $162 \mathrm{bpm}$. After stimulation, mean FHR was 159.44 bpm with a standard deviation of 15.49 and a range of 130 to $187 \mathrm{bpm}(p<0.01)$. Mean RI in the MCA of the fetuses was $75.89 \%$ (range: 64 to 91\%) before the experiment. After the vibro-acoustic stimulation, mean RI was $66.93 \%$ (range: 47 to $83 \%$; $p<0.01$ ).

Conclusions: we observed that a sound stimulus provokes the well-known immediate and significant elevation of FHR and a decrease in cerebral vascular resistance when evaluated by the RI of the fetal middle cerebral artery.

KEY WORDS: Ultrasonography. Fetal physiology. Doppler. Fetal suffering.

\section{Referências}

1. Arey LB. Developmental anatomy. Philadelphia: WB Saunders; 1947.

2. Barden TP, Peltzman P, Graham JT. Human fetal electroencephalographic response to intrauterine acoustic signals. Am J Obstet Gynecol 1968, 100:1128-34.

3. Bast TJ, Anson BJ. The temporal bone and the ear. Springfield: Charles C. Thomas; 1949.

4. Behle I, Lammehirt F, Petracco A, Teixeira J, Rache JE. Valor do teste da estimulação sonora como complemento da cardiotocografia anteparto de repouso. Rev Bras Ginecol Obstet 1982; 4:10414.

5. Oliveira AM, Mauad Filho F, Ruffino Neto A, Gross R, Santana RM, Camano L. Influência do local do estímulo sonoro sobre a resposta acelerativa da freqüência cardiaca fetal. Rev Bras Ginecol Obstet 1982; 3:99-103.

6. Birnholz JC, Benacerraf BR. The development of the human fetal hearing. Science 1983; 222:516-18. 
7. Carrera JM, Mortera C, Torrents M. Doppler en obstetricia. Hemodinamia perinatal. Barcelona: Ediciones Científicas y Técnicas AS; 1992.

8. Carrera JM. Crecimiento Fetal Normal y Patológico. Barcelona: Masson AS; 1997.

9. Clark SL, Sabey P, Jolley K. Nonstress testing with acoustic stimulation and amniotic fluid volume assessment: 5973 tests without unexpected fetal death. Am J Obstet Gynecol 1989; 160:694-7.

10.Dwornicka B, Jasienska A, Smolazrz W, Waldryk R. Attempt of determining the fetal reaction to acoustic stimulation. Acta Otolaryng 1963; 57:571-4.

11.Forbes HS, Forbes HB. Fetal sense reactions: hearing. J Comp Psychol 1927; 7:353-5.

12.Geijn HP, Copray FJA. Procedimientos de control fetal. Barcelona: Masson AS; 1997.

13.Grimwade JC, Walker DW, Bartlett M, Gordon S, Wood C. Human fetal heart rate change and movement in response to sound and vibration. Am J Obstet Gynecol 1971; 109:86-90.

14.Grannum PA, Berkowitz RL, Hobbins JC. The ultrasonic changes in the maturing and their relation to fetal pulmonic maturity. Am J Obstet Gynecol 1979;133:915-22.

15. Hall JG. The cochlea and the cochlear nuclei in neonatal asphyxia. A histological study. Acta Otolaring 1964; 194 (supl):1-93.

16.Hammacher K. Diagnosis of foetal distress by means of cardiotokography (CTG). In: Wood C, Walters WAW, editors. Proceedings of the $15^{\text {th }}$ World Congress Gynecology and Obstetrics. Germany; 1967, 177p.

17.Hon EH. Biophysical studies of the human fetus. In: Diagnosis and treatment of fetal disorders. Adamsons K, editor. New York: Springer-Verlag; 1969.

18.Klosovskii BN. The development of the brain and its disturbance by harmful factors. New York: Pergamon Press; 1963.

19.Luz NP, Luz SH, Lima PC, Feldews VL. Resposta auditiva provocada: nova forma de avaliar as condições fetais. Anais da $23^{a}$ Jornada Brasileira de Ginecologia e Obstetrícia. São Paulo: 1977.

20.Luz NP, Lima CP, Luz SH, Feldens VL. Auditory evoked responses of the human fetus. I. Behavior during progress of labor. Acta Obstet Gynecol Scand 1980;59:395-404.

21.Luzi G, Coata G, Caserta G, Cosmi EV, Di Renzo GC. Doppler velocimetry of the different sections of the fetal middle cerebral artery in relation to perinatal outcome. J Perinat Med 1996; 24: 327-34.

22.Read JA, Miller FC. Fetal heart rate acceleration in response to acoustic stimulation as a measure of fetal well-being. Am J Obstet Gynecol 1977; 129:512-7.

23. Scibetta JJ, Rosen MG, Hochberg CJ, Chik L. Human fetal brain response to sound during labor. Am J Obstet Gynecol 1971; 109:82-5.

24.Smith CV, Phelan JP, Platt LD, Broussard P, Paul RH. Fetal acoustic stimulation testing. II. A randomized comparisons with the nonstress test. Am J Obstet Gynecol 1986; 155:131-4.

25.Sontag LW, Wallace RF. Changes in the rate of the human fetal heart in response to vibratory stimuli. Am J Dis Child 1936; 51:583-9.

26.Sontag LW \& Bernard J. Fetal reactivity to tonal stimulation; a preliminary report. J Gen Psychol 1947; 70:205-10.

27.Veille JC, Penry M. Effects of maternal administration of 3\% carbon dioxide on umbilical artery and fetal renal and middle cerebral artery Doppler waveforms. Am J Obstet Gynecol 1992; 167:1668-71.

28.Visser GA, Muler HH, Wit HP. Vibro acoustic stimulation of the human fetus: effect on behavioural state organization. Early Human Dev 1989; 19:285-96.

29.Zugaib M, Nobile L. Teste da estimulação sônica. I. Proposição de fonte emissora de sons. J Bras Gynecol 1984; 94:113. 